Fecha de recepción: diciembre 2017 Fecha de aceptación: marzo 2018 Versión final: julio 2019

\section{La dialéctica entre lo objetivo y lo vivido: el análisis de la desigualdad social en Córdoba, Argentina.}

Alicia B. Gutiérrez ${ }^{\star}$ y Héctor O. Mansilla ${ }^{\star *}$

Resumen: Uno de los aportes fundamentales de la perspectiva bourdieusiana es su propuesta de una teoría de la práctica como resultado de la relación dialéctica entre sentido objetivo y sentido vivido, lo que implica una toma de posición que es, a la vez, epistemológica, teórica y metodológica. En este texto, explicitaremos primero estos aspectos y luego, en el marco de una investigación sobre la desigualdad social en Córdoba, propondremos una manera de articular ambos sentidos, focalizando en las estrategias educativas y haciendo hincapié en sus consecuencias metodológicas.

Palabras clave: perspectiva bourdieusiana - sentido objetivo - sentido vivido - desigualdad social - estrategias familiares.

[Resúmenes en inglés y portugués en las páginas 108-109]

${ }^{(*)}$ Lic. en Historia (FFyH-UNC), Dr. en Sociologie (EHESS) y Dra. de la Universidad de Buenos Aires-Área Antropología (FFyL-UBA).

Profesora Titular Regular de Sociología (Facultad de Filosofía y Humanidades de la UNC). Investigadora Principal de CONICET. Directora del Programa de Investigaciones "Reproducción social en Córdoba: dinámicas recientes" (CONICET, ANPCyT y UNC). Autora de Las prácticas sociales: Una introducción a Pierre Bourdieu, editado en Argentina y en España (Última edición: EDUVIM, Villa María, 2012). Traductora de libros de Pierre Bourdieu, publicados en Argentina (EUDEBA, Aurelia Rivera, Ferreyra Editor, Siglo XXI) y México (El Colegio de Michoacán). aliciabeatrizgutierrez@gmail.com

${ }^{(*)}$ Doctor en Ciencias Sociales (UBA), Magíster en ciencias sociales -mención en metodología de la investigación social-, Especialista en Diseño de Encuestas y Análisis de Datos y Licenciado en Comunicación Social (UNC). Profesor Asociado de la Cátedra de Análisis de la Comunicación Social II de la Facultad de Ciencias de la Comunicación (UNC), Profesor Asistente en la Cátedra de Metodología de la Investigación Social II de la Facultad de Ciencias Sociales (UNC).

Co-Director del Programa de Investigaciones: "Reproducción social en el Gran Córdoba: dinámicas recientes" (CONICET, ANPCyT y UNC). homansil@hotmail.com 


\section{Introducción: la ontología de la vida social en la perspectiva bourdieusiana y sus consecuencias en el análisis de la desigualdad social}

Cuando Bourdieu hace referencia a la necesidad de superar el "objetivismo" y el "subjetivismo" en las ciencias sociales, y de tomar lo mejor de ambas perspectivas, sus señalamientos suelen ser interpretados sólo como fundamentos de dos momentos analíticos: un primer momento objetivista, en el cual el investigador reconstruye la estructura de relaciones donde se insertan las prácticas, relaciones que son independientes de la conciencia y de la voluntad de los agentes que las producen, y un segundo momento, subjetivista, donde intenta captar representaciones, percepciones y vivencias de esos agentes.

En consecuencia, se toman como dos pasos lógicos del análisis sociológico, que, abordados simultáneamente en una misma investigación, permitirían mayores posibilidades de explicar y comprender la realidad social. Ahora bien, es necesario recordar que esos momentos lógicos se fundan en una ontología particular de la realidad que tomamos como objeto de conocimiento: la vida social existe de doble manera, en las cosas y en los cuerpos, como estructuras objetivas externas y como estructuras objetivas incorporadas. Es más, la vida social no proviene de un contrato explícito entre individuos conscientes y dotados de voluntad, sino que constituye una especie de "orquestación sin director de orquesta" (Bourdieu, 1980a: 99), resultado de una "complicidad ontológica" entre un campo y un habitus (Bourdieu, 1980b: 6). En ese marco, uno de los aportes fundamentales de esta perspectiva es la de recordar permanentemente que toda práctica social tiene dos sentidos: un sentido objetivo y un sentido vivido, y que es necesario que la investigación que llevemos adelante sepa captar el "sentido del juego social", que deviene de la relación dialéctica entre lo objetivo y lo vivido (Bourdieu, 1980a).

Esta propuesta de "teoría de la práctica", desarrollada en detalle en Le sens pratique (Bourdieu, 1980a), conlleva consecuencias epistemológicas, teóricas y metodológicas en todo proceso de investigación. En primer lugar, se trata de una propuesta esencialmente crítica respecto a los límites inherentes a todo conocimiento teórico (opuesto al conocimiento práctico) y pone en cuestión los presupuestos comprometidos en la posición de "observador objetivo" de la realidad social, lo que lleva a analizar la relación subjetiva del científico con el mundo social y con la relación social objetiva que está implicando esta relación subjetiva. En segundo lugar, exige la combinación y articulación de diferentes aproximaciones a la realidad y con ello, la complementariedad adecuada de los métodos cuantitativos y cualitativos: "contra la vieja distinción diltheyana, es necesario plantear que comprender y explicar son una misma cosa" (Bourdieu, 1993: 910).

Así, desde esta manera de concebir la realidad social, analizar las relaciones de desigualdad en un espacio social determinado supone estar en condiciones de dar cuenta de qué manera se distribuyen los recursos (económicos, culturales, sociales, simbólicos), cuáles son las relaciones de fuerza objetivas que sustentan la dominación, y de qué manera estos aspectos son vividos por quienes ocupan las diferentes posiciones sociales. Ahora bien, definidas esas diversas posiciones según el volumen de los recursos familiares y según la estructura particular que conforman (con mayor o menor peso de las diferentes especies de capital disponibles), tenemos que reconocer que, además de "objetivamente" -bajo la forma de propiedades que pueden ser identificadas y medidas con indicadores específi- 
cos-, esos recursos existen también de manera incorporada, bajo la forma de disposiciones a actuar, percibir y valorar.

Avanzando y precisando un poco más, y considerando que en sociedades como las nuestras el recurso más importante es el capital económico (en la medida en que permite caracterizar las grandes clases de condiciones de existencia-Bourdieu,1990-), es importante poder dar cuenta también del peso que tienen los otros recursos en las acumulaciones e inversiones familiares, especialmente el capital cultural ${ }^{1}$ (que permite identificar y caracterizar las fracciones de clase, según la importancia que pudiera tener la Escuela en la reproducción social de la familia). En otras palabras, se trata de estar en condiciones de analizar en qué medida esta especie de capital se pone en juego objetivamente en las diferentes estrategias educativas y en las trayectorias escolares, y de qué modo incide en las maneras en que ellas son percibidas y valoradas como posibles y deseables.

En ese marco de preocupaciones y asumiendo la mirada analítica del estructuralismo constructivista bourdieusiano, nos referiremos a algunos aspectos de la "cocina de la investigación" de un estudio que estamos llevando adelante desde hace varios años, acerca de la reproducción social en Córdoba (Argentina) y de sus dinámicas recientes ${ }^{2}$.

En primer lugar, señalaremos algunas cuestiones teóricas y metodológicas implicadas en nuestra investigación; en segundo lugar, mostraremos brevemente de qué manera captamos el "sentido objetivo" de la problemática que analizamos, identificando diferentes posiciones sociales y en relación con ellas, caracterizando clases y fracciones de clase; luego haremos referencia a la manera como articulamos las dimensiones cuantitativa y cualitativa de nuestra investigación, para, posteriormente, referirnos a los “sentidos vividos" por los agentes que ocupan esas diversas (y opuestas) posiciones en relación con la Escuela, y su lugar en la reproducción social de la familia; finalmente, subrayamos la importancia de esta articulación para dar cuenta del sentido de juego social presente en las estrategias familiares.

\section{El análisis relacional de la desigualdad social en Córdoba, Argentina}

En el marco de nuestro estudio, analizamos un conjunto de prácticas que pertenecen a esferas diferentes (estrategias laborales, habitacionales, educativas y de consumo) y, a través de ellas, estudiamos las maneras como se reproduce la vida social cordobesa y sus relaciones de dominación y de desigualdad.

Partiendo del supuesto de que la vida social se reproduce, como hemos sugerido más arriba, a partir de las prácticas y como resultado de la relación dialéctica entre estructuras y agentes dotados de diferentes grados de poder (Bourdieu 1988 y 1990), tomamos como concepto central el de estrategias de reproducción social, inspirado fundamentalmente en la mirada bourdieusiana. En este sentido, las consideramos como

Conjunto de prácticas fenomenalmente muy diferentes, por medio de las cuales los individuos y las familias tienden, de manera consciente o inconsciente, a conservar o a aumentar su patrimonio, y correlativamente a mantener o mejorar su posición en la estructura de las relaciones de clase (Bourdieu, 1988: 122). 
Dichas estrategias dependen de un conjunto de factores: 1) del volumen y la estructura del capital que hay que reproducir (capital económico, capital cultural, capital social, capital simbólico) y de su trayectoria histórica; 2) del estado del sistema de los instrumentos de reproducción; 3) del estado de la relación de fuerzas entre las clases; y 4) de los habitus incorporados por los agentes sociales.

Estas categorías conceptuales en torno al análisis de las "estrategias de reproducción social" constituyen herramientas fundamentales para analizar la dinámica de las clases en su conjunto, y, con ello, las relaciones de desigualdad social. En efecto, a partir de lo que las diferentes familias ponen en marcha para reproducirse socialmente, estas herramientas muestran claramente una dimensión central en la perspectiva bourdieusiana: la concepción relacional de lo social, herencia estructuralista que queda plasmada en la manera como se construyen los conceptos claves y en el modo como se articulan ${ }^{3}$. Así, las diferentes estrategias de reproducción social se explican sólo relacionalmente, en un doble sentido: en el contexto del sistema que constituyen (en una familia o en un grupo de familias pertenecientes a una clase o fracción de clase) y en el marco más amplio del espacio social global, donde las prácticas y representaciones que forman parte de ese sistema se relacionan con las prácticas y representaciones que son constitutivas de los otros, articulando de esa manera modos de reproducción sociales diferenciales (Gutiérrez, 2004).

De este modo, entendemos el análisis de la desigualdad social en el marco de la estructura de las clases, que también es relacional y que supone la construcción previa del espacio social (Bourdieu, 1990). Por ello, uno de los aspectos más importantes para trabajar, pasa por evitar la sustancialización del concepto de clase, retomando la noción de estructura social para dar cuenta de las propiedades de cada clase que devienen de la pertenencia y ubicación en esa estructura. Así, en tanto conjunto de características posicionales, la clase social deja de ser una sustancia y pasa a ser concebida como una relación, y ello es posible sólo a condición de haber construido el espacio social donde sus propiedades adquieren sentido. (Mansilla, 2017).

En consecuencia, consideramos el "espacio social" como una construcción teórico-metodológica que, tomando simultáneamente un conjunto de variables relativas a recursos económicos y culturales, y apelando a métodos específicos, nos permite dar cuenta de la estructura de las relaciones de desigualdad, caracterizar las diferentes posiciones sociales, e identificar clases y fracciones de clase. Esas posiciones diferentes, junto al volumen y la estructura del capital que las definen, constituyen principios fundamentales para poder explicar y comprender las estrategias de reproducción social, junto a las condiciones objetivas que caracterizan los diferentes ámbitos de las prácticas (trabajo, educación, vivienda, etc.) y las disposiciones incorporadas de quienes las producen.

Nuestra investigación implica una articulación teórico-metodológica que contempla tres etapas analíticas, con un diseño relacional que vincula un conjunto de técnicas para la construcción del espacio social, la caracterización de las clases y la selección de casos para el armado de la muestra de la fase cualitativa.

1) La primera etapa es fundamentalmente cuantitativa, donde proponemos una construcción relacional de las clases y fracciones del Gran Córdoba, a partir del conjunto de recursos -fundamentalmente económicos y culturales- que disponen las familias para su reproducción; es decir, se trata de construir el sentido objetivo de nuestra problemática. 
Para ello, apelamos a la utilización de métodos de estadística descriptiva multidimensional conforme a la escuela francesa de análisis de datos, aplicando de manera conjunta métodos factoriales y de clasificación, tomando como base la información captada por la "Encuesta Permanente de Hogares" (EPH) en los terceros trimestres de los años 2003, 2005, 2007, 2009, 2011, 2013 y 2015, y complementándola con informaciones derivadas de otras fuentes, tales como la Encuesta Nacional sobre Acceso y Uso de Tecnologías de la Información y Comunicación (ENTIC) de 2011, la Encuesta Nacional de Gastos de Hogares (ENGHo) 2012/2013 y la Encuesta Nacional sobre la Estructura Social (ENES) implementada en el marco del Programa de Investigación sobre la Sociedad Argentina Contemporánea (PISAC). En esta primera etapa hemos propuesto una construcción del espacio social cordobés ${ }^{4}$, identificando y caracterizando relacionalmente clases y fracciones de clase y describiendo las regularidades presentes, tanto en lo que llamamos "instrumentos de reproducción social" (especialmente mercado laboral, escolar, habitacional y de las políticas públicas) cuanto de las prácticas asociadas a las estrategias de reproducción social de las familias cordobesas. (Gutiérrez y Mansilla, 2016).

2) A partir de estos resultados, la segunda etapa consiste en analizar las prácticas, interacciones y representaciones concretas de las estrategias y del modo como se articulan con las diferentes posibilidades y límites relativos a las condiciones de existencia de cada clase y fracciones de clase. Se trata de un segundo momento de carácter subjetivista, que apunta a captar los sentidos vividos y atribuidos por los propios agentes, a las prácticas y a las condiciones en las que se despliegan. Este segundo momento contempló entonces, la realización de diferentes entrevistas en profundidad a casos seleccionados a partir de las características asociadas a cada clase y fracción (contemplando su peso relativo) construidas en la primera etapa, además de observaciones e historias de vida, para captar trayectorias individuales y familiares representativas de cada una de ellas.

3) Una tercera etapa, implica la puesta en relación de las diferentes condiciones de existencia relevadas particularmente en la primera etapa (volumen y estructura de capital e instrumentos de reproducción social disponibles), con los sentidos vividos y prácticas concretas. A partir de ello, elaboraremos "tipologías de sistemas de estrategias de reproducción” representativas de las diferentes posiciones en el espacio social que constituye el Gran Córdoba. En definitiva, nuestra propuesta analítica de la desigualdad social intenta recuperar las dimensiones estructural e histórica y toma relacionalmente tanto las estrategias y representaciones cuanto las condiciones objetivas que las hacen posibles (sentidos vividos y sentidos objetivos).

\section{Del sentido objetivo al sentido vivido: articulación de las dimensiones cuantitativa y cualitativa}

En otros lugares hemos presentado la construcción del espacio social cordobés, junto al conjunto de discusiones teóricas y decisiones metodológicas y técnicas que derivan de la necesidad de adecuar nuestra perspectiva teórica y metodológica a la disponibilidad de información, y que aquí sólo mencionamos brevemente (Gutiérrez y Mansilla, 2015 y 2016; Mansilla, 2017). 
Propusimos una construcción relacional de las clases y fracciones de clase cordobesas, tomando en cuenta el conjunto de recursos -fundamentalmente económicos y culturalesque disponen las familias para su reproducción. Con un software específico (SPAD 5.0), aplicamos de manera conjunta un análisis de correspondencias múltiples (ACM) y una clasificación jerárquica ascendente (CJA) tomando como base la Encuesta Permanente de Hogares $^{5}(\mathrm{EPH})$, como hemos mencionado más arriba.

Esta encuesta ofrece dos bases: hogares e individuos. Como nuestra unidad de análisis es la familia, considerada como una unidad doméstica de producción y de consumo, hemos combinado ambas bases. De este modo, seleccionamos propiedades referidas al capital económico y cultural de nuestras unidades de análisis, que corresponden tanto al hogar como a su referente de hogar (RH). Y ello porque esta persona de referencia, por su posición dominante en el grupo familiar, transfiere sus recursos a través de las relaciones de distribución que constituyen a la familia como sujeto colectivo.

Así, en relación con la disponibilidad de capital económico del hogar, se tomó en cuenta en primer lugar, el ingreso total individual del $\mathrm{RH}$, que incluye los ingresos por su ocupación principal, así como cualquier otro -rentas, herencias, etc.-. Si bien esta variable funciona como uno de los principales indicadores económicos de la familia, se seleccionó también el ingreso per cápita familiar (IPCF), lo que permitió capturar todos los recursos económicos del grupo familiar y ponerlos en relación con la cantidad de miembros. Ambas variables dan cuenta de la capacidad económica de la familia.

Como indicador de capital cultural fue seleccionado el máximo nivel de instrucción formal alcanzado por el RH (capital escolar como subespecie del capital cultural). Fueron considerados otros indicadores que remiten tanto al capital económico como al cultural, en particular aquellos vinculados a la inserción del RH en las relaciones de producción, como la jerarquía laboral (en tanto indicador de la distribución desigual de la autoridad en el campo laboral) y la calificación ocupacional (como indicador de competencias y conocimientos asociados al puesto de trabajo). Por último, se tomaron también como variables activas el sexo, edad y situación conyugal del $\mathrm{RH}$, como elementos claves de diferenciación social que remiten a ciertas formas de capturar el ciclo vital familiar. Estas ocho variables activas (51 modalidades) nos permitieron construir los espacios sociales cordobeses, mientras que el resto de las variables de la EPH fueron consideradas como ilustrativas.

Nuestro objetivo en este apartado es presentar el modo en que operamos la articulación de las etapas cuantitativa y cualitativa. Tal articulación supuso, en el plano técnico, un proceso de selección de agentes para la realización de entrevistas. Para ello, consideramos las principales propiedades constitutivas de las clases construidas, a fin de dar cuenta de los esquemas interpretativos y de los sentidos vividos, en relación con la posición y trayectoria social, en tanto inversiones en los distintos dominios de las prácticas. Así, se tornó necesario mantener el perfil de estos grupos en la selección de los entrevistados para poder establecer las comparaciones que el análisis relacional requiere. Para dicha selección utilizamos los resultados de la construcción del espacio social de 2011, y la caracterización de sus clases (Gráficos 1 y 2 ). 


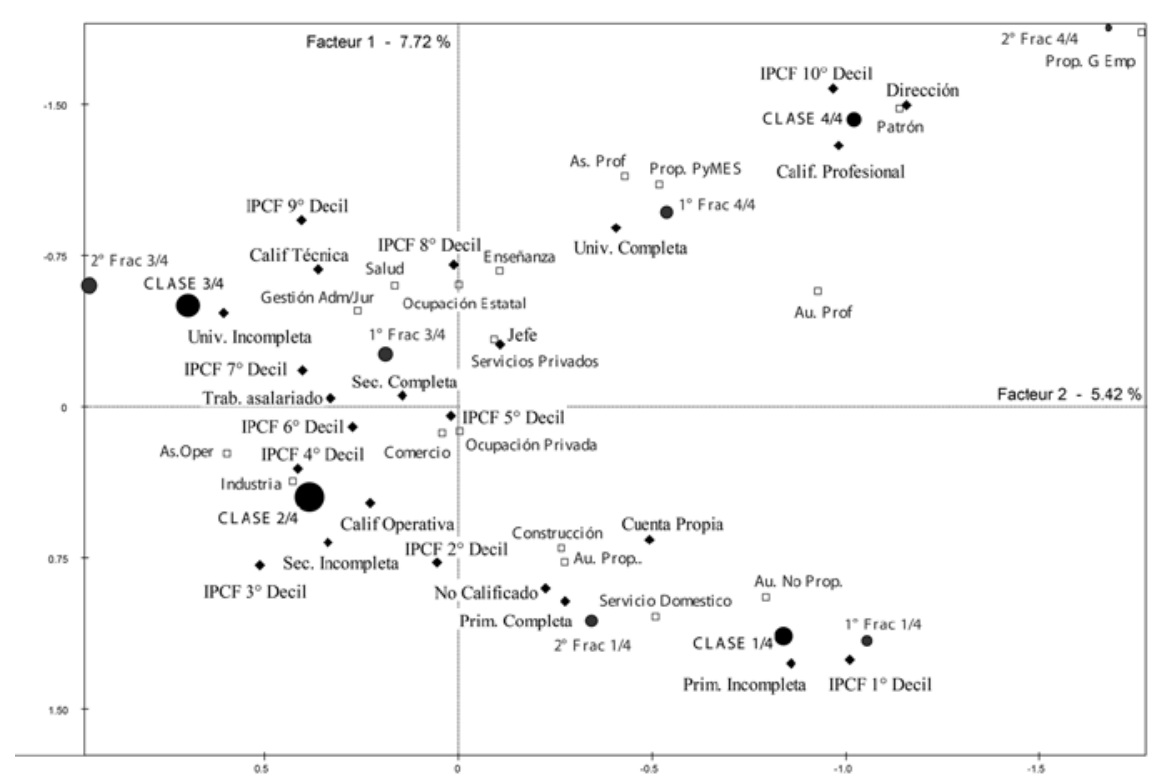

Gráfico 1. El espacio social cordobés 2011 en el plano de los ejes 1 y 2 (13,14\% de inercia y 51 modalidades activas).

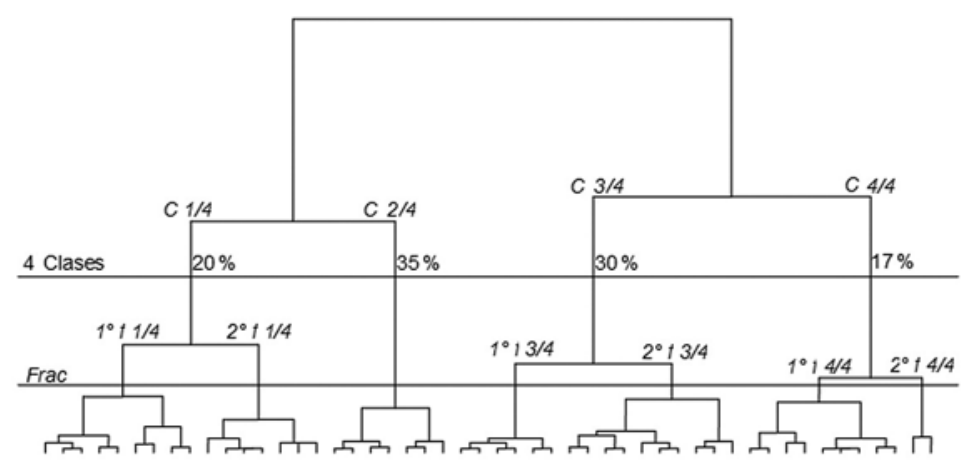

Gráfico 2. Espacio Social Córdoba 2011 - Dendrograma de la CJA para los primeros 4 factores (22,69\% de inercia). Particiones para 4 clases y 6 fracciones. 
Para evitar un análisis de la desigualdad social en términos sustancialistas, es necesario proceder de manera relacional tanto en la construcción del espacio social cuanto en su descripción: en este sentido, no tomamos cada grupo de familias de manera aislada, sino que reconstruimos el sistema de relaciones a partir del cual los definimos y caracterizamos, desde proximidades y distancias en el espacio social.

Así, es posible ver que las diferencias que se establecen entre las grandes clases sociales, y que a su vez las constituyen como tales, remiten a relaciones de desigualdad en torno a aspectos centrales de la reproducción económica de las familias, en particular la inserción laboral de sus referentes, y los recursos que entran en juego en esas prácticas. Es decir, aquí el peso fundamental lo tiene el capital económico, y, en menor medida, el capital cultural, especialmente en su subespecie escolar.

La región del cuadrante inferior derecho del espacio agrupa posiciones ocupadas por familias en las que el referente de hogar vende su fuerza de trabajo, y lo hace a través de un modo de inserción en el mercado laboral que se caracteriza por no requerir calificación alguna, no percibir aportes ni beneficios sociales y por obtener bajas remuneraciones. Estas posiciones poseen muy bajo volumen patrimonial tanto en lo económico como en lo cultural, aunque la característica diferencial que articula su relación con las demás posiciones es la venta de fuerza de trabajo sin calificación y en situaciones de precariedad laboral. Por su parte, las familias que se ubican en las regiones medias dominadas se caracterizan por vender fuerza de trabajo de baja o media calificación: este aspecto los diferencia tanto de la clase anterior como de las otras clases medias que, ubicadas en mejores posiciones sociales, venden fuerza de trabajo de mayor calificación. Notamos en este caso la importancia fundamental del capital cultural acumulado, en la medida en que mejores puestos en el mercado de trabajo se encuentran en relación con los más altos niveles educativos de sus referentes, lo que repercute directamente en los ingresos que perciben.

Por otra parte, es posible que las diferencias entre los cuadrantes superiores (izquierdo y derecho) respondan tanto a la división social del trabajo, vinculada a la propiedad-no propiedad de medios de producción, como a la división técnica en el proceso de trabajo, entre quienes ejecutan tareas y quienes las dirigen y controlan. Mientras los referentes del cuadrante superior izquierdo (clase media dominante) desarrollan una inserción laboral basada en la venta de fuerza de trabajo altamente calificada, vinculada a ocupaciones estatales en ramas de la educación y la salud, que articulan dicha inserción con su capital cultural (capital escolar asociado a formación universitaria completa), los referentes del cuadrante superior derecho presentan asociaciones con cargos y funciones directivas. A su vez, éstos son en su mayoría compradores de fuerza de trabajo, a diferencia de los otros, que la venden. Ahora bien, con relación al capital económico expresado por los IPCF y los ingresos individuales del referente, hay que tener en cuenta que, si bien los datos muestran que la clase alta dominante posee ingresos que se ubican en el $10^{\circ}$ decil, este tramo de los ingresos per cápita es muy amplio (oscila entre \$5000 y \$30000 -entre 1163 y 6977 dólares-), a diferencia del $9^{\circ}$ decil de la clase media dominante, que implica ingresos que van de $\$ 3300$ a $\$ 4900$ (de 767 a 1140 dólares), lo que puede esconder profundas desigualdades entre ambos grupos y al interior de la clase alta dominante ${ }^{6}$. Así, podemos suponer que los ingresos de los asalariados estatales de la clase media dominante (limitados en sus ingre- 
sos) pueden encontrarse muy por debajo de los de las familias con referentes propietarios de empresas o asalariados profesionales directivos en la esfera pública o privada.

Esto nos permite observar la presencia de lo que se denomina una estructura en quiasma. Es decir, una estructura que, a lo largo del factor que expresa el volumen global de capital, diferencia aquellas regiones donde es posible observar cierta primacía del capital cultural sobre el económico (clases y fracciones que articulan sus estrategias de reproducción a partir de la potencialidad de sus recursos culturales y que generalmente se encuentran ubicadas en la región izquierda del espacio) de aquellas otras donde se observa la primacía del capital económico en las inversiones que definen sus estrategias de reproducción (ubicadas sobre la región derecha del espacio social). En este caso, si bien podemos estar hablando de volúmenes de capital relativamente iguales, en el sector izquierdo del espacio se identifica la zona donde el peso de la reproducción social está asociado a las acumulaciones e inversiones de y en capital cultural, lo que además sugiere hipotéticamente en cuáles ámbitos sociales se incentivan las prácticas: el sistema escolar en general, y los distintos mecanismos a partir de los cuales se acumula ese capital.

Teniendo en cuenta estos aspectos, presentaremos ahora las características específicas de cada una de las clases y fracciones de clase que hemos identificado, junto a la selección de los entrevistados pertinentes, a fin de captar los sentidos vividos de esas posiciones objetivas.

\section{1. Clase baja (20\%)}

Estas familias se caracterizan por su bajo volumen global de capital, con una estructura patrimonial asociada a bajos ingresos: IPCF en el $1^{\circ}$ decil, ingreso total del $\mathrm{RH}$, ingreso por ocupación principal del $\mathrm{RH}$ e ingreso total familiar en el $1^{\circ}$ decil. En lo que respecta a la ocupación de su RH se asocian a la ausencia de calificación laboral, al servicio doméstico, al cuentapropismo y a la construcción, con cierta precariedad en el trabajo: escasa antigüedad laboral en pequeños establecimientos del ámbito privado, o como trabajadores autónomos y sin cobertura médica. El capital escolar del RH es relativamente escaso y va de nivel primario incompleto al primario completo. Respecto a las características de hogares y viviendas de esta clase, puede observarse una asociación a $\mathrm{RH}$ femeninos, de edad mayor, hogares con problemas de hacinamiento y condición de ocupante de la vivienda como régimen de tenencia. Por último, puede señalarse que esta clase de familias se asocia a la recepción de subsidios y ayuda material.

III. 1. 1. Primera fracción (10\%): Familias que poseen en un alto porcentaje (casi el $80 \%$ ) un RH femenino. Se trata de mujeres separadas o viudas, sin calificación laboral y que se desempeñan en el servicio doméstico y cuidado de personas. 


\begin{tabular}{|c|l|c|}
\hline Entrevistado & \multicolumn{1}{|c|}{ Descripción } & Total \\
\hline Carolina & $\begin{array}{l}\text { Trabajadora sin calificación en la rama del empleo doméstico. Edad: } 41 \\
\text { años. Nivel educativo: secundario incompleto. Hogar de tres miembros }\end{array}$ & \\
\hline Noemí & $\begin{array}{l}\text { Trabajadora sin calificación en el cuidado de personas. Edad: } 69 \text { años. } \\
\text { Nivel educativo: primario incompleto. Hogar de nueve miembros }\end{array}$ & 3 \\
\hline Mirna & $\begin{array}{l}\text { Trabajadora autónoma sin calificación -costurera-. Edad: } 62 \text { años. Nivel } \\
\text { educativo: primario completo. Hogar de 3 miembros }\end{array}$ & \\
\hline
\end{tabular}

III. 1. 2. Segunda fracción (12\%): Hogares que en su mayoría poseen un RH masculino, vinculado a la construcción y al cuentapropismo, con calificación laboral operativa, y que conforman hogares numerosos.

\begin{tabular}{|c|l|c|}
\hline Entrevistado & & Total \\
\hline Guillermo & $\begin{array}{l}\text { Albañil. Edad: } 51 \text { años. Nivel educativo: primario completo. } \\
\text { Hogar de 6 miembros }\end{array}$ & \multicolumn{1}{|c|}{ Descripción } \\
\hline Danilo & $\begin{array}{l}\text { Pintor de obra. Edad: 55 años. Nivel educativo: secundario completo. } \\
\text { Hogar de seis miembros }\end{array}$ & 6 \\
\hline Arturo & $\begin{array}{l}\text { Albañil. Edad: 27 años. Nivel educativo: secundario incompleto. } \\
\text { Hogar de 8 miembros }\end{array}$ & $\begin{array}{l}\text { Albañil. Edad: 44 años. Nivel educativo: primario completo. } \\
\text { Hogar unipersonal, recientemente separado, anteriormente de 7 } \\
\text { miembros }\end{array}$ \\
\hline Darío & $\begin{array}{l}\text { Jardinero. Edad: 34 años. Nivel educativo: secundario incompleto. } \\
\text { Hogar de 5 miembros }\end{array}$ \\
\hline Rafael & $\begin{array}{l}\text { Carpintero. Edad: 29 años. Nivel educativo: secundario completo. } \\
\text { Hogar de 4 miembros }\end{array}$ \\
\hline
\end{tabular}

\section{2. Clase media dominada (35\%)}

Se trata de posiciones con un volumen global medio-bajo de recursos. Las familias pertenecientes a esta clase poseen una estructura patrimonial asociada principalmente a un IPCF que va del $3^{\circ}$ al $5^{\circ}$ decil. Aunque los ingresos del RH, tanto por su ocupación principal como sus ingresos totales se ubican en deciles más altos, el elevado número de miembros del hogar tiende a disminuir su IPCF. La clase posee características del RH asociadas principalmente a calificaciones laborales operativas, en la industria y la logística, con capital cultural relativamente más alto que en la clase anterior, medido por niveles de instrucción ubicados en los estudios secundarios incompletos. A estas características se suman otras con un menor nivel de asociación, que vinculan a los RH con el cuentapro- 
pismo, la construcción y la administración pública. Si bien es una clase sin fracciones es posible recortar dos grupos a su interior fundamentalmente por la edad del RH y la rama de actividad. Una primera fracción (menos numerosa) se asocia a referentes de mayor edad (+50) vinculados al cuentapropismo y a la logística desde hace más de cinco años. La segunda (algo más numerosa) parece estar compuesta por referentes más jóvenes ( 35 a 50 años) vinculados a la industria y con hogares numerosos.

\section{2. 1. Primera fracción (16\%)}

\begin{tabular}{|c|l|c|}
\hline Entrevistado & \multicolumn{1}{|c|}{ Descripción } & Total \\
\hline Laura & $\begin{array}{l}\text { Empleada. Edad: } 64 \text { años. Nivel educativo: secundario completo. } \\
\text { Hogar de dos miembros }\end{array}$ & \multirow{2}{*}{6} \\
\hline Eduardo & $\begin{array}{l}\text { Mecánico autónomo. Edad: } 60 \text { años. Nivel educativo: secundario } \\
\text { incompleto. Hogar de tres miembros }\end{array}$ & \\
\hline Iván & $\begin{array}{l}\text { Taxista. Edad: } 66 \text { años. Nivel educativo: secundario completo. } \\
\text { Hogar de dos miembros. }\end{array}$ & \\
\cline { 1 - 2 } Milo & $\begin{array}{l}\text { Jardinero. Edad: } 64 \text { años. Nivel educativo: secundario incompleto. } \\
\text { Hogar de dos miembros }\end{array}$ & $\begin{array}{l}\text { Taxista. Edad: } 61 \text { años. Nivel Educativo: secundario completo. } \\
\text { Hogar de dos miembros }\end{array}$ \\
\hline Alberto & $\begin{array}{l}\text { Camionero. Edad: } 55 \text { años. Nivel Educativo: secundario incompleto. } \\
\text { Hogar de dos miembros }\end{array}$ & \\
\hline
\end{tabular}

\section{2. 2. Segunda fracción (19\%)}

\begin{tabular}{|c|c|c|}
\hline Entrevistado & Descripción & Total \\
\hline Carina & $\begin{array}{l}\text { Operaria fábrica automotriz. Edad: } 41 \text { años. Nivel educativo: secundario } \\
\text { completo. Hogar de cuatro miembros }\end{array}$ & \multirow{6}{*}{6} \\
\hline Norberto & $\begin{array}{l}\text { Operario fábrica automotriz. Edad: } 47 \text { años. Nivel educativo: primario } \\
\text { completo. Hogar de } 6 \text { miembros }\end{array}$ & \\
\hline Fermín & $\begin{array}{l}\text { Operario fábrica automotriz. Edad: } 43 \text { años. Nivel educativo: secundario } \\
\text { completo. Hogar de cuatro miembros }\end{array}$ & \\
\hline Adam & $\begin{array}{l}\text { Chofer transporte interurbano. Edad: } 31 \text { años. Nivel educativo: } \\
\text { universitario incompleto. Hogar de } 4 \text { miembros }\end{array}$ & \\
\hline José & $\begin{array}{l}\text { Empleado fábrica de sándwiches. Edad: } 43 \text { años. Nivel educativo: } \\
\text { universitario incompleto. Hogar de cinco miembros }\end{array}$ & \\
\hline Marina & $\begin{array}{l}\text { Empleado en fábrica de cerámicos. Edad: } 34 \text { años. Nivel educativo: } \\
\text { terciario no universitario, incompleto. Hogar de cuatro miembros }\end{array}$ & \\
\hline
\end{tabular}




\section{3. Clase media dominante (29\%)}

Estas familias se caracterizan por poseer RH jóvenes que alcanzan, en su mayoría, una calificación ocupacional técnica, estudios superiores universitarios incompletos o completos y un IPCF ubicado entre el $7^{\circ}$ y el $9^{\circ}$ decil, con ocupaciones asociadas a los servicios sociales, en particular a la educación, la gestión administrativa y la salud. Se trataría de una clase que desarrolla sus estrategias poniendo en juego capital cultural, aprovechando a su favor especialmente ciertos mecanismos de objetivación de su capital escolar y sus instancias de legitimación en el mercado laboral. Otras características vinculadas a esta clase muestran hogares unipersonales o pocos numerosos, con RH jóvenes, en su mayoría mujeres, solteras y sin presencia de menores de diez años.

III. 3. 1. Primera fracción (15\%): Familias que presentan una fuerte asociación con RH femeninos, quienes, con estudios universitarios completos, han acumulado mayor capital cultural que su fracción vecina. Invierten este recurso en estrategias laborales basadas en la ocupación de puestos asociados a las esferas de la educación y la salud, preferentemente en el ámbito estatal.

\begin{tabular}{|c|l|c|}
\hline Entrevistado & \multicolumn{1}{|c|}{ Descripción } & Total \\
\hline Sandra & $\begin{array}{l}\text { Docente de nivel medio. Edad: } 50 \text { años. Nivel educativo: terciario } \\
\text { completo. Hogar de tres miembros }\end{array}$ & \multirow{2}{*}{5} \\
\cline { 1 - 2 } Lucía & $\begin{array}{l}\text { Médica ginecóloga. Edad: } 30 \text { años. Nivel educativo: universitario } \\
\text { completo. Hogar unipersonal }\end{array}$ & \\
\cline { 1 - 2 } Luisa & $\begin{array}{l}\text { Vicedirectora de escuela de nivel medio. Edad: } 57 \text { años. Nivel educativo: } \\
\text { universitario completo. Hogar unipersonal }\end{array}$ & $\begin{array}{l}\text { Médica clínica. Edad: } 54 \text { años. Nivel educativo: universitario completo. } \\
\text { Hogar de tres miembros }\end{array}$ \\
\hline Viviana & $\begin{array}{l}\text { Empleada pública como ingeniera en sistemas. Edad: } 43 \text { años. Nivel } \\
\text { educativo: universitario completos. Hogar unipersonal }\end{array}$ & \\
\hline
\end{tabular}

III. 3. 2. Segunda fracción (18\%): Fracción asociada a RH jóvenes, solteros, que conforman hogares unipersonales, con trabajos asalariados, de calificación técnica, en la gestión administrativa y jurídica.

\begin{tabular}{|c|l|c|}
\hline Entrevistado & \multicolumn{1}{|c|}{ Descripción } & Total \\
\hline Enrique & $\begin{array}{l}\text { Empleado administrativo (vendedor). Edad: 33 años. Nivel educativo: } \\
\text { terciario no universitario completo. Hogar de 3 miembros }\end{array}$ & \\
\hline Nahuel & $\begin{array}{l}\text { Empleado administrativo. Edad: 25 años. Nivel educativo: universitario } \\
\text { incompleto. Hogar unipersonal }\end{array}$ & \\
\hline
\end{tabular}




\begin{tabular}{|c|l|c|}
\hline Flavio & $\begin{array}{l}\text { Empleado administrativo. Edad: 32 años. Nivel educativo: universitario } \\
\text { incompleto. Hogar de dos miembros }\end{array}$ & \\
\hline Gerardo & $\begin{array}{l}\text { Programador de HP. Edad: 41 años. Nivel Educativo: universitario } \\
\text { completo. Hogar de dos miembros }\end{array}$ & \multirow{2}{*}{8} \\
\hline Iris & $\begin{array}{l}\text { Empleada en la Secretaría de Cultura del Gobierno Provincial. Edad: } 36 \\
\text { años. Nivel educativo: universitario incompleto (en curso). Hogar de dos } \\
\text { miembros }\end{array}$ & \\
\hline Jorgelina & $\begin{array}{l}\text { Empleada de embotelladora Coca Cola. Edad: } 26 \text { años. Nivel educativo: } \\
\text { universitario incompleto (en curso). Hogar de dos miembros }\end{array}$ & \\
\hline Pedro & $\begin{array}{l}\text { Preceptor en escuela secundaria. Edad: 32 años. Nivel educativo: } \\
\text { terciario no universitario incompleto. Hogar unipersonal }\end{array}$ \\
\hline Dante & $\begin{array}{l}\text { Empleado de comercio. Edad: 32 años. Nivel educativo: universitario } \\
\text { incompleto. Hogar unipersonal }\end{array}$ \\
\hline
\end{tabular}

\section{4. Clase alta dominante (17\%)}

Está compuesta por familias que poseen un alto volumen global de capital, tanto económico como cultural. Su patrimonio se encuentra asociado a ingresos que se ubican en el $10^{\circ}$ decil (ingreso total del RH, Ingreso por ocupación principal, IPCF e ITF). Poseen RH con calificación ocupacional profesional, que ocupan puestos directivos y realizan dicha función. Son patrones o propietarios tanto de grandes empresas como de Pymes y poseen un nivel de instrucción superior universitario. Esta clase se caracteriza por RH que son en su mayoría varones, se asocian a ocupaciones del Estado, en la rama de la educación, y sus viviendas poseen buenas condiciones habitacionales.

III. 4. 1. Primera fracción (13\%): Fracción compuesta por un 13\% de hogares que, no estando asociados a $\mathrm{RH}$ patrones o propietarios, muestran que su estructura patrimonial es fundamentalmente cultural. Estas posiciones están asociadas a un elevado capital escolar, lo que se complementa con la ocupación de trabajos asalariados de categoría profesional en el ámbito del Estado, en la enseñanza, operando sistemas y equipos informáticos y con cargos directivos, todos ellos indicadores del control del proceso de trabajo en su división técnica.

\begin{tabular}{|c|l|c|}
\hline Entrevistado & \multicolumn{1}{|c|}{ Descripción } & Total \\
\hline Andrea & $\begin{array}{l}\text { Directora de instituto terciario. Edad: } 58 \text { años. Nivel educativo: } \\
\text { universitario completo. Hogar unipersonal }\end{array}$ & \\
\hline Federico & $\begin{array}{l}\text { Director de escuela secundaria y docente. Edad: } 52 \text { años. Nivel } \\
\text { educativo: posgrado universitario. Hogar de cuatro miembros }\end{array}$ & \\
\cline { 1 - 2 } Magdalena & $\begin{array}{l}\text { Asesora técnica. Edad: } 57 \text { años. Nivel educativo: posgrado universitario. } \\
\text { Hogar unipersonal }\end{array}$ & \\
\hline
\end{tabular}




\begin{tabular}{|c|l|c|}
\hline José & $\begin{array}{l}\text { Abogado, prosecretario de justicia. Edad: } 46 \text { años. Nivel educativo: } \\
\text { universitario completo. Hogar de cinco miembros }\end{array}$ & \multirow{2}{*}{6} \\
\hline Silvana & $\begin{array}{l}\text { Abogada, jueza de cámara. Edad: } 60 \text { años. Nivel educativo: posgrado } \\
\text { universitario. Hogar de cuatro miembros. }\end{array}$ & \\
\hline Ricardo & $\begin{array}{l}\text { Investigador de CONICET. Edad: } 61 \text { años. Nivel educativo: posgrado } \\
\text { universitario. Hogar de dos miembros }\end{array}$ & \\
\hline
\end{tabular}

III. 4. 2. Segunda fracción (5\%): Fracción más pequeña, que presenta fuertes asociaciones con indicadores de propiedad de empresas y $\mathrm{RH}$ patrones, ocupando cargos de dirección o jefatura fundamentalmente en el ámbito privado.

\begin{tabular}{|c|l|c|}
\hline Entrevistado & \multicolumn{1}{|c|}{ Descripción } & Total \\
\hline Esteban & $\begin{array}{l}\text { Dueño de gran empresa. Edad: } 65 \text { años. Nivel educativo: universitario } \\
\text { incompleto. Hogar de tres miembros }\end{array}$ & 2 \\
\cline { 1 - 2 } Mariano & $\begin{array}{l}\text { Dueño de gran empresa y asesor. Edad: } 57 \text { años. Nivel educativo: } \\
\text { posgrado universitario. Hogar de dos miembros }\end{array}$ & \\
\hline
\end{tabular}

En consecuencia, seleccionamos un total de 43 referentes de hogar (RH) de diferentes clases y fracciones de clase, teniendo en cuenta que los recursos económicos y culturales que poseyeran pudieran ser representativos de los perfiles característicos de cada una de ellas. En otras palabras, buscamos una variación en las condiciones de producción de sus discursos que fuera consistente con el establecimiento de las distintas condiciones objetivas (clase y fracción), junto a su peso relativo, que identificamos y caracterizamos en el espacio social en la primera etapa. De esta manera, estuvimos en condiciones de trabajar con un campo de discursos producidos en diferentes condiciones, susceptibles de ser puestos en relación y sometidos a un análisis comparativo.

\section{Posiciones y tomas de posición respecto a la Escuela y las estrategias escolares}

El campo escolar es un universo fundamental en la reproducción de las relaciones de poder y de desigualdad en un espacio social concreto y situado en el marco de las relaciones capitalistas contemporáneas. En ese sentido, para asegurarse la reproducción (o la mejora) de la posición social, las familias tienden, de manera consciente o no consciente, a realizar inversiones (dinero, tiempo, ayudas de todo tipo) en estrategias escolares diversas, que serán más importantes y exigirán más esfuerzos, cuanto más importante sea el capital cultural en la composición del patrimonio de la familia y cuanto mayor sea su peso relativo respecto al capital económico. 
En ese contexto, podemos decir que, para comprender una estructura objetiva de clases, como la que hemos descripto brevemente para la ciudad de Córdoba, Argentina, debemos considerar también la dimensión subjetiva de esa estructura. A modo de ejemplo, nos focalizaremos ahora en algunos aspectos de las inversiones escolares de las familias, en el papel que le asignan a la Escuela y en las valoraciones y representaciones de las trayectorias posibles, todas ellas dimensiones de los sentidos vividos asociados a posiciones diferenciales en una estructura de desigualdad.

Para mostrar el análisis del conjunto de nuestras entrevistas, veamos a continuación cuatro maneras diferentes de percibir y valorar la escuela, resultado de cuatro posiciones diferentes (y opuestas) en el espacio social cordobés: una, ligada a las condiciones objetivas de la clase baja dominada, con escaso volumen de capital económico y cultural, la otra, asociada a la clase alta dominante, precisamente a la fracción más rica en capital cultural, y otras dos, vinculadas a las clases medias (dominada y dominante), con diferentes capitales culturales relativos, aunque con tomas de posición semejantes.

\section{1. Clase baja dominada: bajo registro de las trayectorias escolares y escasa valoración de la escuela}

Danilo es un representante típico de la clase baja y con hijos de diferentes edades. Nació y creció como hijo único en un barrio pobre de la ciudad. Tiene 55 años y su madre era empleada doméstica. Es el referente de un hogar conformado por él, su esposa de 43 años, y cuatro hijos. La familia vive en una casa sencilla, que heredaron hace dos años por el fallecimiento de la madre del $\mathrm{RH}$ y de su segundo esposo, quien sería el propietario original. Danilo es pintor de obra desde los 12 años y hace changas, siempre autónomamente: lo contratan los vecinos y algunos comerciantes de la zona.

Una frase que repite varias veces en la entrevista es: "No me acuerdo, realmente no me acuerdo". El bajo registro de la trayectoria educativa de su familia, expresado en el desconocimiento de los estudios de sus padres e incluso, aún más próximo en el tiempo, de los de sus hijos mayores, mantiene una relación inteligible con el hecho de que para él sus estudios no le sirvieron "para nada, porque al final sigo trabajando".

E- ¿Danilo terminó sus estudios...?

D- Ni a palos... no terminó ni una cosa ni la otra. Nada.

E- ¿La primaria tampoco?

D- La primaria sí. Igual que el Agustín.

E- ¿Y se acuerda en qué año abandonó Agustín?

D- No me acuerdo, realmente no me acuerdo.

Este bajo registro va acompañado de representaciones y valoraciones que privilegian la cercanía física de los establecimientos educativos, como el elemento determinante de la elección de las instituciones escolares para sus hijos:

D: “[A la escuela primaria] la elegimos porque estábamos viviendo cerca...Y cuando pasaron a la secundaria estaban ahí nomás, a cinco cuadras para adentro estaba el otro colegio. No me acuerdo si era... IPET N ${ }^{\circ} 26$, algo así era”. 
Sin embargo, quizás en parte por la imposición de la temática educativa en la entrevista, Danilo manifiesta sus expectativas sobre la educación de sus hijos menores, que aún asisten a la escuela primaria. El propio tono de resignación y el anclaje a unas posibilidades objetivas se encuentran inscriptos en unas disposiciones que lo llevan a esperar "que lleguen a algo el día de mañana, que no anden burreando como uno".

Con un volumen de capital escaso, tanto en términos económicos como culturales, las familias posicionadas en el sector más dominado del espacio social se caracterizan también por inversiones escolares que tienden, a lo sumo, a alcanzar los estudios primarios. En todo caso, la trayectoria escolar futura se percibe como un anhelo difuso ("que lleguen a algo el día de mañana"), más que como un aliciente claro tendiente a las inversiones reales, fundamentalmente porque la Escuela "no sirve para nada" en los puestos de trabajo disponibles para ellos.

\section{IV.2. Clase alta con predominancia de capital cultural: inversiones y trayectoria marcada por la Escuela}

José, con un perfil típico de la primera fracción de la clase alta, es abogado y ha realizado la carrera judicial (actualmente es Prosecretario de la rama penal). Nació en Río Cuarto y migró a la ciudad de Córdoba para estudiar Derecho. Tiene 46 años y está casado con María, que es bióloga. Tienen tres hijas, dos de las cuales están cursando estudios secundarios. Además de su casa en un barrio típico de las clases dominantes, poseen un campo en las sierras, que han destinado a una especie de refugio donde tienen crianza de diversos animales. Por el lado de su familia política, su suegro es juez y todos sus cuñados son profesionales. Por su parte, su padre hizo carrera en la Fuerza Aérea, y su madre en Tribunales como empleada administrativa. Tiene dos hermanas: una diseñadora gráfica, la otra con estudios de Derecho incompletos.

La estructura patrimonial de la familia de José está dominada claramente por el capital cultural, y por ello, reclama de fuertes inversiones educativas para mantener o mejorar su posición. Primero de tres hijos, su migración a Córdoba significó un gran esfuerzo de sus padres, en un momento de fuerte crisis hiperinflacionaria en Argentina: "no había departamentos para alquilar disponibles, entonces bueno, ahí empezó (...) un periplo y una serie de mudanzas. Primero vivía en una casa con otros ocho estudiantes mayores que yo, mucho más grandes...”.

A diferencia de la unidimensionalidad puesta en juego por la familia de Danilo a la hora de buscar Escuela (la distancia física), José comenta que él y su esposa consideraron varios aspectos que ellos tomaban como fundamentales, y que la elección de la institución para las hijas, estuvo signada por un cálculo explícito en términos de costos y beneficios:

"Hicimos un... casting de escuelas. Un casting de escuelas. Queríamos una escuela que no fuera privada en un cien por ciento, que fuera mixta, por cierto, que fueran varones y mujeres... eh... que estuviese cerca, de casa, y bueno, que a nosotros nos constatara, a través de la vivencia de otros padres, que hubiese un buen nivel educativo. Y pasando por todo ese tamiz, nos quedamos con la que más se aproximaba a lo que nosotros queríamos 
(...) Hicimos como una averiguación así general, incluso conocimos a un par de docentes, o padres que estaban mandando los chicos a ese colegio..."

Tanto José como su mujer valoran su participación en la educación de sus hijas, de lo que se sienten orgullosos: “...Sí, María participa un montón, recomprometida (...) Va a reuniones, se mantiene en mucho contacto con las otras madres, mucho contacto con los docentes".

A su vez, destaca y valora la disposición que han adquirido sus hijas sobre su propia formación: “...Rocío, me dice... 'Papá, que hay cosas de matemática que no entiendo, qué sé yo’, y está yendo a una maestra particular de matemáticas (...) Ella misma, previniendo, se prepara... no, son muy responsables".

En el caso de José y su familia, con la dedicación puesta tanto en la elección de la Escuela para las hijas, como en el desarrollo de sus actividades educativas y la participación parental en ellas y a nivel de la institución, podemos observar claramente un conjunto de inversiones en tiempo, en esfuerzo, en dinero, típica de una fracción de clase que se reproduce reproduciendo el capital cultural, fundamentalmente escolar.

\section{3. Las clases medias: diferentes trayectorias escolares, igual valoración positiva de la escuela y apuestas por el sector privado}

\section{3. 1. Clase media dominada: educación privada para un mejor trabajo}

Norberto se ubica en el perfil que corresponde a la clase media dominada (segunda fracción). Hombre de 47 años, cuyo máximo nivel de estudios es primario completo; Silvia, su cónyuge de 44 años, posee estudios secundarios incompletos, cursados en una escuela pública y es ama de casa. Él es empleado en la fábrica de camiones IVECO, que pertenece al grupo FIAT (sector industrial). Con una antigüedad de 28 años en la misma fábrica, opera máquinas y equipos electrónicos. La familia se completa con cuatro hijos.

Ni la familia de Norberto ni la de Silvia han acumulado ni se han valido mucho del capital escolar: de sus numerosos hermanos y cuñados sólo uno terminó el secundario. A su vez, los recursos económicos también han sido escasos, lo que se traduce en una trayectoria de clase que los ubica en posiciones dominadas del espacio social. Valoran el trabajo, pero también la educación como posibilidad de mejora laboral y de vida en general. Al respecto dicen:

"S: Bueno.... a mí, lo que por ahí siento que lo poco que estudié, es lo que me da fuerza ahora para educar a mis hijos. Que ellos terminen. A pesar que no han terminado, hago el sacrificio que sea, para que ellos se reciban de algo. Más que todo por el trabajo, porque yo he trabajado en casas de familia y eso. Pero quiero que ellos no... No porque sea feo, sino porque yo quiero que no sea tan sacrificada la vida de ellos.

$\mathrm{N}$ : yo también pienso lo mismo... aparte bueno... yo hice primaria.... por ahí hay cosas ahora..."

Y en el valor positivo asignado a la educación, se evidencia una diferencia simbólica entre el sector privado y el público, que justifica sus inversiones, especialmente económicas:

"En el caso de Giselle, que es la primera nena que tuvimos (...) fue a la primaria acá en el barrio, y ya la secundaria fue a un colegio católico y privado" (...). Es lo que yo por ahí rescato de los colegios privados...de que... rescato y comparo con mis sobrinos, así... de 
que a un colegio privado, es como que ya al alumno ya lo van mentalizando de que tiene que seguir una carrera. Yo por ahí veo con los colegios públicos, es como que no hacen mucho hincapié, los profesores, en que hay que esforzarse, en que hay que seguir... El Leonel el año que viene ya pasaría directamente a la Facultad y ya los mismos profesores, lo van encaminando, diciéndole...en que lo ven que le gustaría más, o qué.... Digamos, lo veo más que lo incentivan". (...) "Uno hace mucho sacrificio para pagarle digamos el colegio Renault a él. Pero uno ya ve los frutos de que... va a seguir, en cambio, si lo mandamos a un público.... yo sé que cuando termina el público, más que trabajar de albañil o una changa, otra cosa no..., es muy difícil".

Con relativamente bajo volumen de capital económico y cultural (aunque en cantidad superior a las de clase baja), esta familia realiza inversiones importantes en la Escuela, "muchos sacrificios" que permiten delinear una trayectoria escolar marcada por la incorporación de habilidades técnicas de la misma rama de actividad que pone en juego el padre, pero con perspectivas mayores de perfeccionamiento, sobre todo para el hijo varón. Así, Leonel va a una escuela secundaria técnica prestigiosa y privada (el Instituto Renault) y se prepara para ingresar a la Universidad, lo que le permite decir a su madre: "hago el sacrificio que sea para que ellos se reciban de algo".

\section{3. 2. Clase media dominante: educación privada, "buena base para todo".}

Luisa representa el perfil de la primera fracción de la clase media dominante. Con 54 años, es la mayor de tres hermanos. Nació en la ciudad de Córdoba y desde su nacimiento vivió sobre el kilómetro 6 y 1/2, camino a Monte Cristo, donde la familia tiene una quinta que heredó de su abuelo paterno. Cursó sus estudios primarios y secundarios en colegios privados, mientras que sus dos hermanos menores asistieron a colegios públicos. Por otra parte, es la única en la familia que logró completar sus estudios universitarios. Se recibió de médica y realizó su residencia en San Francisco, Córdoba. Luego, se trasladó al sur del país, donde trabajó un año, pero por problemas de salud regresó a Córdoba. Se casó a los 28 años y se trasladó a la localidad de Comechingones, donde tuvo a sus dos hijas: Ayelén de 20 años, estudiante de psicología en la UNC, y Julieta, estudiante de nivel medio de 18 años. Con padres que no terminaron los estudios primarios (tercer y cuarto grado) y hermanos que asistieron a escuelas públicas sin culminar sus estudios secundarios, en el caso de Luisa, y tal vez por ser la primera de los hijos, su familia realizó una fuerte apuesta en su educación: "Yo fui a un colegio privado, San María Antonia, en barrio Yofre Norte... que es un colegio católico privado. Hice primario y secundario. Y los chicos [sus hermanos] hicieron en el colegio público... porque yo creo que mis padres (...) priorizaban la educación, la buena educación. Entonces como yo era la más grande era como que ellos me mandaron... porque, por ejemplo, estaba la abuela Ema y mis tíos me apoyaron para que yo hiciera los primeros años en esa escuela privada. Después, quizás, a los más chicos también les hubieran hecho lo mismo, pero ya no tenían más capacidad, no podían... (...) No tenían recursos ni económicos, ni... económicos vendría ser... Es decir, como que a mí con mucho esfuerzo lo pudieron hacer, pero para ellos no alcanzó, definitivamente".

Así como valora su elección vocacional, destaca el valor de la educación que obtuvo e intenta transmitir a sus hijas esta disposición hacia el estudio: 
"Y bueno con respecto a lo cultural a todo y sí. Cambia totalmente el que se egresa de una universidad, de cualquier universidad, en relación al que no lo tiene, yo creo que vale la pena el esfuerzo, aunque no sean grandes los beneficios económicos, yo creo que sí”.

Y si los beneficios económicos le permiten sostener su posición con dificultad, esta situación parece sólo acentuar las disposiciones hacia las inversiones educativas como modo de reproducir su lugar, y realizar el destino probable de sus hijas:

"Yo siempre apuesto, aunque en mi vida no fue tan fructífero, por supuesto. Yo a las chicas les inculco que es importantísimo. Yo creo que ellas también lo saben, estudiar... ¡bah! formarse de una u otra forma. Sí."

Y justifica la elección de un colegio privado para ellas: "porque uno priorizaba, bueno en un tiempo no se pudo, pero uno priorizaba que tuvieran una buena formación, sí... una buena base... qué sé yo, para todo".

Luisa muestra una trayectoria escolar diferente a las de sus padres e incluso a las de sus hermanos menores: estudios primarios y secundarios en una escuela privada y estudios universitarios completos. Se trata de una acumulación importante de capital cultural, asociada a la educación privada como garantía de "una buena formación”, que abre la puerta a los estudios universitarios y al sostenimiento de una trayectoria escolar valorada positivamente, más allá del diploma como capital cultural institucionalizado: "cambia totalmente el que egresa de una Universidad", "yo siempre apuesto (...) a las chicas les inculco que es importantísimo".

\section{A modo de cierre}

La vida social como resultado de la dialéctica entre el sentido objetivo y el sentido vivido, constituye uno de los aportes fundamentales de la sociología de Bourdieu. Asumiendo esta perspectiva, hemos presentado de qué manera abordamos la desigualdad social en Córdoba, haciendo hincapié, fundamentalmente, en sus consecuencias metodológicas.

La lógica de pensamiento relacional de la mirada bourdieusiana obliga a asumir una estrategia de investigación que permita la puesta en relación de las diferentes condiciones sociales de existencia de las familias cordobesas, con los sentidos vividos sobre el conjunto de prácticas que constituyen sus estrategias de reproducción social. A través del análisis de estos sentidos vividos buscamos llegar a los esquemas de acción y percepción que los agentes ponen en juego en estas prácticas: su "sentido práctico", sentido adquirido mediante un proceso de familiarización en el mismo espacio y tiempo -socialmente estructurados- en que los agentes son producidos, sentido ajustado a las divisiones sociales en las que se hallan inscriptos.

En ese marco, hemos mostrado de qué manera construimos el espacio social cordobés, como una estructura de posiciones definidas a partir del volumen y la estructura del capital que poseen las familias (especialmente de capital económico y de capital cultural y sus pesos relativos), lo que hace visibles las relaciones de desigualdad objetiva entre ellas, conformando diferentes clases y fracciones de clase. Ahora bien, esta estructura de desigualdad objetiva cobra su verdadera importancia cuando estamos en condiciones de analizar la dinámica de su reproducción; es decir, cuando abordamos prácticas, interacciones, 
representaciones y valoraciones que constituyen la dimensión subjetiva de esas relaciones de desigualdad.

Una manera de captar aquel sentido del juego social que propone Bourdieu, como resultado de la relación dialéctica entre el sentido objetivo y el sentido vivido (Bourdieu, 1980), consiste en articular ambas dimensiones, tras la hipótesis de que se manifiestan en estructuras homólogas. Así, a partir de los perfiles definidos por nuestro análisis, para cada clase y fracción de clase, y teniendo en cuenta el peso relativo de cada una de ellas en la estructura del espacio social, seleccionamos un conjunto de 43 referentes de hogar, con los cuales pusimos en marcha la etapa cualitativa de nuestro estudio.

A modo de ejemplo de nuestra manera de proceder en el análisis, mostramos, en el marco de nuestro esquema general de selección de casos representativos (por perfil y por cantidad) del espacio social cordobés, 4 maneras diferentes de representarse, valorar y actuar en relación con las estrategias escolares y las trayectorias recorridas, y/o, incluso, vividas como posibles y pensables, que forman parte de modos de reproducción social diferentes. En efecto, en sociedades como las nuestras, no sólo es importante la acumulación del capital económico, también lo es la inversión en capital cultural (bajo sus diferentes formas, pero sobre todo bajo la subespecie de capital escolar) y, de hecho, la acumulación diferencial de ambas especies (y su peso relativo) nos permite visualizar cuál de ellas es objeto de las mayores inversiones, y cómo cada modo de reproducción le concede a la Escuela un lugar diferente.

Así mostramos cómo un bajo volumen de capital económico y cultural, representativo de las posiciones más dominadas del espacio social, se asocia a bajos registros de los caminos recorridos por los miembros de la familia en el sistema escolar y a una escasa valoración de la Escuela respecto a los beneficios que otorga, concentrando los esfuerzos del grupo en los aspectos ligados más directamente a la reproducción material. En la posición objetiva opuesta, hemos visto cómo un alto volumen de capital económico y cultural, con predominancia de este último en la estructura, hace que todos los miembros de la familia inviertan en su acumulación y cuidado: en la elección deliberada y pensada del tipo de establecimiento de los hijos y en el esfuerzo económico y en el tiempo dedicado, haciendo de la Escuela un ámbito privilegiado de su modo de reproducción social. En las posiciones medias del espacio social (con un volumen medio del capital económico y cultural), y desde dos contextos de acumulación de capital cultural y de trayectorias diferentes (una de relativamente escaso capital escolar, la otra de una trayectoria que completa estudios universitarios) encontramos una alta valoración de la Escuela (y fundamentalmente del sector privado como garantía de formación) y una visualización clara de trayectorias probables para los hijos, marcadas por el sistema escolar.

Todo ello nos recuerda que cada agente adquiere sus competencias sociales en el espacio social en el que se inscriben sus prácticas, y en su desarrollo interioriza la estructura social desde su propia posición. Así, espacio social y habitus están organizados según los mismos principios. Se trata de lógicas de acción y relaciones de fuerzas que, incorporadas, estructuran el sentido práctico de cada agente determinando desde el propio lugar que éste ocupa, lo indecible, lo impensable y lo realizable para él o para su grupo. Es a partir de este sentido práctico que los agentes distinguen lo relevante, ponen de relieve lo importante frente a lo superfluo y dan sentido así a prácticas propias y ajenas. 
Por ello, es necesario subrayar que nuestra perspectiva de estudio asume el discurso de los agentes (sobre sus prácticas y sus representaciones) como una práctica más. Y como tal, ese discurso es producto de unas disposiciones (disposiciones a nombrar, a hacer decible, a valorar) determinadas por la posición del agente en el espacio social. De este modo, su análisis debe devolver al enunciado toda su materialidad: poner al discurso en relación con sus condiciones de producción para buscar regularidades discursivas, para situar cada enunciado en un campo de enunciados posibles y determinar, mediante la comparación entre estos, las presencias y ausencias significativas. Así, contra la idea de que los discursos son entes que contienen en sí su sentido, este tipo de análisis toma como punto de partida su proceso y el lugar social de su producción. En consecuencia, no se analizan los enunciados en sí mismos sino en relación con sus condiciones de producción, a fin de dar cuenta de los esquemas interpretativos que están en su base-aquellos esquemas que son comunes a todos los miembros de una clase- para resituar el análisis en un campo de comparación (Martín Criado, 1998).

Desde la propuesta bourdieusiana, nuestra investigación se sostiene, como hemos descripto, en dos momentos analíticos y en su articulación lógica. Sólo a partir de la construcción del espacio de las clases y fracciones de clase, estuvimos en condiciones de identificar agentes representativos de cada una de ellas para diseñar un conjunto de entrevistas en profundidad. Esto nos permitió no sólo poner en relación los discursos relevados con sus condiciones de producción, sino también determinar y comparar lo decible de cada uno, variando las condiciones de producción que están en su base. Esta variación resultó imprescindible para poder establecer la incidencia de las condiciones de producción en la estructuración de los discursos.

De esa manera, pudimos abordar también la dimensión subjetiva de la desigualdad social, como expresión del modo en que se perciben las diferentes posibilidades que se presentan para actuar, de la manera en que se valoran los diferentes campos de acción (el trabajo, la Educación, etc.) y de las formas que adoptan según sistematizaciones y jerarquizaciones que responden a trayectorias individuales, familiares y de clase, cuestiones que, sin duda, constituyen un aspecto fundamental de las relaciones de poder en un espacio social determinado.

\section{Notas}

1. El capital social y el capital simbólico, por su parte, funcionan como especies de sobreañadidos de los otros dos, que pueden potenciar su rendimiento diferencial en los distintos ámbitos en que se ponen en juego.

2. Se trata de un Programa de Investigaciones llamado "Estrategias de reproducción social en familias cordobesas: dinámicas recientes", dirigido por Alicia B. Gutiérrez y Héctor O. Mansilla y radicado en Córdoba. Cuenta con financiamiento del Consejo Nacional de Investigaciones Científicas y Técnicas (CONICET), el Fondo para la Investigación Científica y Tecnológica (FONCYT) y de la Secretaría de Ciencia y Técnica (SECyT) de la Universidad Nacional de Córdoba.

3. Hemos desarrollado en otro lugar la dimensión relacional de estos conceptos para el análisis de las situaciones de pobreza y de desigualdad social (Gutiérrez, 2007). 
4. En este sentido, una primera propuesta puede verse en Gutiérrez y Mansilla, 2013, y una segunda, corregida y ampliada en Gutiérrez y Mansilla, 2015.

5. La EPH es un programa nacional de captura permanente de datos demográficos y socioeconómicos de la población, vinculadas a la fuerza de trabajo. Se realiza trimestralmente, tiene cobertura nacional y abarca los mayores centros urbanos del país. Elegimos el tercer trimestre por la posibilidad de relacionar nuestros resultados con los otros relevamientos continuos que se realizan en ese mismo período.

6. Como referencia, consideremos que en ese momento $1 U \$ A$ equivalía a $4,30 \$$ y que el salario mínimo, vital y móvil se ubicaba en los 2.300 -535 dólares, aproximadamente-.

\section{Referencias bibliográficas:}

Bourdieu, P. (1980a). Le sens pratique, Paris: Seuil.

Bourdieu, P. (1980b). “Le mort saisit le vif. Les relations entre l'histoire réifiée et l'histoire incorporée”, Actes de la recherche en sciences sociales, 32-33, pp. 3-14.

Bourdieu, P. (1988) [1979]. La Distinción: Criterios y bases sociales del gusto, Madrid: Taurus.

Bourdieu, P. (1990) [1984]. "Espacio social y génesis de las clases", Sociología y Cultura, México: Grijalbo, pp. 281-310.

Bourdieu, P (1993). "Comprendre", in La misère du monde, Paris: Seuil, pp. 903-939.

Gutiérrez, A. (2004). Pobre’ como siempre... Estrategias de reproducción social en la pobreza, Córdoba: Ferreyra Editor.

Gutiérrez, A. (2007). "Herramientas teórico-metodológicas de un análisis relacional para los estudios de la pobreza", Ciencia, Docencia y Tecnología, 35, pp. 15-33.

Gutiérrez, A. y Mansilla, H. (2013). "El espacio social y su reproducción: aspectos teórico metodológicos y fuentes secundarias", XXIX Congreso de la Asociación Latinoamericana de Sociología -ALAS- 2013 "Crisis y Emergencias Sociales en América Latina”, Santiago de Chile.

Gutiérrez, A. y Mansilla, H. (2015). “Clases y reproducción social: el espacio social cordobés en la última década”, Política y sociedad, 52, 2, pp. 409-444.

Gutiérrez, A. y Mansilla, H. (2016). El espacio social de las clases y los instrumentos de reproducción social, Córdoba: Universidad Nacional de Córdoba, 2016.

Mansilla, H. (2017). El consumo de Tecnologías de Información y Comunicación (TIC) en las estrategias de reproducción social de los hogares cordobeses. Tesis de Doctorado, UBA. Martín Criado, E. (1998). Producir la Juventud, Madrid: Akal.

\footnotetext{
Abstract: One of the fundamental contributions of the Bourdieusian perspective is its proposal of a theory of practice as a result of the dialectical relation between objective meaning and experiential meaning, which implies a position taking that is, at the same time, epistemological, theoretical and methodological. In this text, we will first explain these aspects and then, within the framework of a research on social inequality in Cór-
} 
doba, we will propose a way to articulate both senses, focusing on educational strategies and emphasizing their methodological consequences.

Key words: bourdieusian perspective - objective meaning - experiential meaning - social inequality - family strategies.

Resumo: Uma das contribuições fundamentais da perspectiva bourdieusiana é a proposta de uma teoria da prática como resultado da relação dialética entre significado objetivo e significado vivido, o que implica uma tomada de posição que é, ao mesmo tempo, epistemológica, teórica e metodológica. Neste texto, vamos primeiro explicar esses aspectos e, em seguida, no âmbito de uma pesquisa sobre a desigualdade social em Córdoba, vamos propor uma maneira de articular os dois sentidos, com foco em estratégias educacionais e enfatizando suas conseqüências metodológicas.

Palavras chave: perspectiva bourdieusiana - significado objetivo - significado vivido - desigualdade social - estratégias familiares.

[Las traducciones de los abstracts fueron supervisadas por el autor de cada artículo] 